\title{
Using participatory research methods to explore residents' perception of workplace stressors during residency training
}

\author{
Fauzia N. Minai ${ }^{1}$, Syeda K Ali ${ }^{2}$
}

\begin{abstract}
Introduction: The culture of the learning environment is a major determinant of behaviours developed by residency trainees. This study explores anaesthesia residents perceptions of workplace stressors which affect behaviour and work performance adversely during the training period.

Methods: We used two complementary qualitative participatory research methods based on focus group discussions and semi-structured interviews, with volunteers from each year of residency. The discussions were held in complete privacy and recorded on a digital voice recorder. Report of each discussion was shared with all participants to ensure credibility through member checking.
\end{abstract}

Results: The stressors identified by anaesthesia residents which impaired work performance and generated stress were related to multiple supervisors, conflicting attitudes of different supervisors, workload and unsupportive organizational culture outside the parent department.

Conclusion: Tensions due to lack of tolerance for divergent practices, diverse faculty, lack of constructive feedback and respect are the major stressors identified by residents which affect behaviour adversely. This information is a valuable resource for evaluation of workplace culture as perceived by the trainees, and planning self-development programs for faculty members and medicals resident.

Keywords: stress in residency, resident support, professionalism, organizational culture

\section{Introduction}

Residency training is a stressful period (Martini et al, 2004; Samuel et al, 1991; Ratanawongsa et al., 2008; Ratanawongsa et al., 2007). All trainees do not perform equally inspite of uniformity of academic selection criteria (McCaul et al., 2007; Jordan et al., 2005) because there is a shift from classroom learning to experiential workplace learning requiring specific behavioural skills. The organizational

\footnotetext{
${ }^{1} \mathrm{Dr}$, FCPS, Anaesthesiology,

Assistant Professor,

Department of Anaesthesia

Aga Khan University

${ }^{2} \mathrm{Dr}, \mathrm{MHPE}, \mathrm{Ph} \mathrm{D}$

Associate Professor,

Department of Educational Development

Aga Khan University

Corresponding author:

Dr. FauziaMinai

Assistant Professor,

Department of Anaesthesia, Aga Khan University,

Stadium Road, P.O Box 3500

Karachi 74800, Pakistan

Email: fauzia.minai@aku.edu
}

culture or 'unstated' curriculum is an important factor in shaping behaviour of trainees during workplace learning (West \& Shanafelt, 2007; Thomas, 2004; Nyssen et al., 2003). The ACGME has incorporated professional behaviours as an exit level competency in every training program since 2007 and medical residents' physical as well as emotional well-being is a major concern in the development of professionalism (Ratanawongsa et al., 2008; Ratanawongsa et al., 2007, Marieniss, 2004; Klein et al., 2003; Radhika et al., 2006). We chose to explore the medical residents' perceptions of workplace stressors which affect their behaviour, work performance and academic achievement adversely, in our own training program. Our objective was to gain insight into the remediable factors in the learning environment which would facilitate academic achievement and professional behaviours in the trainees.

Educators have used qualitative, quantitative and mixed method approaches to assess students' perception in relation to the educational environment (Tobin et al., 1998). Although Maslach Burnout Inventory (MBI) and Beck Depression inventories enable 
students' perceptions of their educational environment to be quantified and compared, (Martini et al. 2004; Samuel et al., 1991; Jordan et al., 2005; Nyssen et al., 2003; Marieniss, 2004; Roff et al., 1997; Al-Hazimi et al., 2004; Khan, 2008) these study designs require larger number of subjects for reliable and valid interpretations of the data (Frazer, 1998). The researchers are able to gain insight into group data and obtain information on the overall trends. A review of literature (Thomas, 2004) showed that most studies examining burnout dimensions among residents used survey questionnaires and inventories as the main data source. A qualitative study on the other hand, may be used to gather information regarding the research problem with respect to the beliefs, feelings, opinions and perceptions from a smaller group (Ratanawongsa et al., 2008; Gay et al., 2012). Focus groups discussions were useful and contributed to that understanding (Liamputtong, 2006).

\section{Methods}

We considered that exploration of perceptions, experiences and responses of residents to workplace stressors would yield subjective information, which would need verification, probing and validation. This data could not be collected by quantitative methods. We chose two complementary qualitative research methods to facilitate our objective (Liamputtong, 2006). We decided that Focus Group Discussions with a moderator who is not a member of the residency committee would generate confidence for disclosures through interaction among a group of residents, maximize data yield by the 'chaining' or 'cascading' effect, and facilitate verification of extracted data by all participants. We also believed that semi structured group interviews based on themes verified by the entire group would enable in depth exploration of themes without any ambiguity, and concurrence would ensure maximum credibility of the information.

This study was conducted at the Department of Anaesthesia, Aga Khan University after approval from the University Ethics Review committee.

\section{Process Participants}

Through purposive sampling we recruited 2-4 anaesthesia residents from residency year 1 (R1) to residency year 5 (R5) to participate in the study. Our goal was to target a diverse mix of residents currently in training at each stage of residency. Voluntary participations were invited through email and direct contact. The junior and senior residents representatives were briefed about the study to facilitate residents availability and were also recruited as participants because they are in touch with all ongoing residents' issues.

\section{Participants' profile}

The participant's age range was 28-35 years. Six residents from year 2 and 3 and nine residents from year 4 and 5 volunteered. None of the residents from year 1 volunteered to participate possibly due to uncertainty or perceived repercussions. Six residents were unmarried and nine were married. Only one out of four female residents volunteered to participate. All participants were graduates of Pakistani medical colleges. Language proficiency in Urdu and English was similar although the participants belonged to diverse ethnic groups.

\section{Profile of investigators}

Fauzia Minai is an Assistant Professor with 15 years in the same organization in various positions. She is involved in departmental and university undergraduate and postgraduate education at Aga Khan University.

Syeda Kauser Ali is a Senior Lecturer at the Department for Educational Development at Aga Khan University. She is involved in university undergraduate and postgraduate education. She has experience in faculty development, curriculum development and assessments.

Venue was secured in advance ensuring complete privacy and written informed consent was taken from all participants. Participants were asked to complete a form requesting relevant demographic information. Fifteen of the thirty residents in the anesthesia program participated in the study. Since participation was voluntary we did not consider gender balance or marital status in the group composition. The first author conducted the sessions.

The author initially reassured the participants about confidentiality and anonymity in any dissemination of information to put the participants at ease. She then discussed the definition and perception of stress with participants to obtain agreement on the concept of negative and positive stress (Appendix 1). When agreement was obtained participants were asked to describe what they thought of as negative stressors in the 
workplace. The discussion was then led by open ended questions and probing based on emerging themes which were used as interview prompts (Appendix 2). Five focus groups discussion of 90 minutes to two hours duration were held between July to September 2008 until "thematic saturation" was achieved, that is discussions were yielding confirmatory rather than new themes. Recording was done on a digital voice recorder and transcribed by a transcriber recruited from the medical records department. Confidentiality of the data and security of the recorded discussion was maintained by the principal investigator, coinvestigator and transcriber.

\section{Analysis}

The tapes and the transcribed notes were reviewed by the first author for correctness and completion. Data was then analyzed manually and coded independently by the two investigators. Differences in coding were discussed and a consensus agreement reached, where needed. Similar coding was then merged in order to identify emerging themes. These were then reviewed for agreements by both the investigators. Verification of themes identified from each discussion was sought from participants after each transcription prior to subsequent discussions. The completed analysis of the focus group discussions was sent to the participants of the focus group discussions thus ensuring credibility through member checking. The report was found to be satisfactory by the participants and no amendments were suggested.

\section{Results}

The major themes which emerged as a source of negative stress can be broadly classified into those caused by factors within the department and those outside the department. The stressors within the department of anesthesia are grouped into those that are related to supervision, specialty demands, workload and resident support, while outside the department these are perceived to be due to organizational culture and institutional policies (Table 1). The specific stressors as identified by the residents under these themes and subthemes are given below.

\section{A. Within Anesthesia Department}

\section{a. Supervision related stressors}

i. Multiple supervisors and expectations gap: Residents are stressed by inability to understand the expectations of a very diverse faculty, and their ability level especially in the early years of training. This expectations gap is made worse by lack of communication and discussion of learning objectives at the start of rotations. As one of the resident quoted "the first discussions should not be like viva examinations".

ii. Lack of constructive feedback: The residents feel threatened by fear of adverse remarks over questions or answers. They also feel that their learning is inhibited by negative criticism and lack of constructive feedback. According to a senior resident "When a consultant disagrees with our plan we are not told what is wrong with the plan or what is right". This generates uncertainty and anxiety. Another resident said that "we need a friendly environment to discuss things openly."

iii. Lack of tolerance for mistakes: The residents feel pressured to get things right the first time which they feel is an unjustified expectation. Quote "the way mistakes are handled is stressful and not conducive to learning" and another "there should be instruction and advice rather than rebuke".

iv. Lack of control over learning and participation in decisions: The residents feel that lack of control over their learning also affects performance negatively and those that give them this control is very helpful. However, this is seldom done and leads to stress of inadequate performance. "Some consultants ask what do you want to discuss and this is very good practice".

v. Inflexible attitudes and wide variations in practice of consultants: This inhibits open discussions and development of a thought process in problem solving and eventually affects development of critical thinking ability.

vi. Lack of appreciation and encouragement: Over a period of time, this is demotivating, and affects the drive to do their best. "We are criticized when something is wrong but never appreciated when everything is right."

\section{b. Work related stressors}

Residents said that they feel harassed at being pressured with time at handing over one case and taking in of new cases especially by paramedical staff and surgeons, along with the 
burden of punctilious documentation. Unrealistic lists with pressure to continue after allotted time is a stress faced by senior residents doing lists with minimal supervision. Dealing with dictation by surgeons affects work performance and attitudes adversely. Multitasking by placement in several areas of service, distant supervision in locations outside operating rooms and anticipation of sudden events add to stress of performance and learning. Work and home balancing is very difficult because of long hours and academic deadlines. Coping with these multiple stresses tends to cause fatigue and burnout.

\section{c. Residents support related stressors}

Residents asserted that they feel that there is a lack of resident support and their say in closure of loops in incident reports. "Personal contacts and backdoor reporting by faculty and paramedical staff is used as a weapon to threaten residents."

"One sided decisions are taken without residents' feedback".

"When we have no reason to expect it we suddenly find negative comments written in our file". "Even when we air our problems nothing is done about it. No visible change occurs".

Residents asserted that "Policies and procedures should not be bypassed". Residents also feel that mentors are strongly needed in the program because supervisors provide dissertation supervision only. "Their role is very limited and the Departmental Residency Committee cannot solve every individual's problem". They also said that that they should have control the over nominating their representative to the DRC. "Residents representatives are nominated by DRC chair. They should be nominated by residents".

\section{B. Outside Department of Anaesthesia}

\section{a. Organizational Culture Related}

Residents desire bonding and easy relationships with colleagues at all levels. "We should be like a family." They feel their performance is affected adversely by a gap of shared goals, understanding and trust between multiple care providers necessarily involved in anesthesia care. "It is highly stressful to deal with an emergency or critical situation where to some documentation and administrative protocols are more important than the crisis at hand. Lack of team work, cooperation and collaboration is very frustrating and hinders smooth clinical work". In locations outside main operating rooms stress of distant supervision, dismissive and disrespectful attitude of other consultants and nurses causes intense performance anxiety.

"Residents of all levels should be respected and supported as learners instead of being dismissed as junior or inferior". The lack of respect leads to a lot of frustration and demotivation, affecting performance in senior years where increasing responsibilities are added but environment is unsupportive. The learner's response is anger and aggressiveness. Development of professional behaviour, empathy and advocacy for the patient is overshadowed by the adaptive behaviours developed for their own survival.

\section{b. Institutional policies related}

Resource constraints drive residents to work extra hours. Some factors identified include deficiency of accommodation close to premises for residents, no transport arrangements for residents in case of strikes and unrest in the city which is frequent, insufficient time for orientation given to new inductees. These factors, stretched over long working hours, and five years of residency affect their performance, academic achievement, personal lives and personalities adversely, particularly in junior years.

\section{Discussion}

The learning environment or the unstated curriculum plays an important role in shaping behaviours and attitudes in trainees. It is what the resident perceives as "the way things are done." Hence, awareness of the residents perceptions is important. An environment unsupportive for the learner affects negatively mental and emotional wellbeing, which is reflected in defensive behaviours and impaired work performance. Discussion, dialogue and openness between faculty and learner fosters a non-threatening non-judge mental learner friendly environment which is conducive to attainment of academic goals with professional behaviours (West \& Shafelt 2007). Inflexible attitudes and lack of tolerance for different practice modalities among a very diverse faculty creates confusion in the learner. A culture of tolerance, respect and encouragement fosters the same values in the learner and provides motivation for excellence (Tobin \& Fraser, 1998). Faculty development courses which provide insight into the importance of the formal and unstated curriculum are essential for supervision of trainees. The coping skills of residents would also be strengthened by short courses on self- 
Table 1: Classification of stresses caused by factors within the department and outside the department

\begin{tabular}{|c|c|c|}
\hline Major Themes & Subthemes & Specific stressors \\
\hline \multicolumn{3}{|c|}{ A Within the department of anesthesia } \\
\hline & \multirow[t]{6}{*}{ Supervision related } & Lack of specific feedback if disagree with plan developed \\
\hline & & Different styles of consultants \\
\hline & & Lack of appreciation \\
\hline & & Expectations not communicated at the start of rotation \\
\hline & & Gap of expectations between residents and faculty \\
\hline & & $\begin{array}{l}\text { Inhibition in asking questions openly because of fear of } \\
\text { rebuke }\end{array}$ \\
\hline & \multirow[t]{4}{*}{ Specialty related } & Anticipation of sudden events \\
\hline & & Surges of stress \\
\hline & & Distant supervision in outside operation theatre services \\
\hline & & Long hours \\
\hline & \multirow[t]{4}{*}{ Workload related } & No timings for handing /taking over of new cases \\
\hline & & Multitasking \\
\hline & & Long lists of cases (in appropriate time expectations) \\
\hline & & No time for home/children \\
\hline & Resident Support related & No backup/support from faculty in contentious matters \\
\hline \multicolumn{3}{|c|}{ B Outside the department of anesthesia } \\
\hline & \multirow[t]{6}{*}{ Organizational culture } & Lack of respect by URs and Nurses \\
\hline & & $\begin{array}{l}\text { Lack of trust by residents (mostly in surgery and OBGYN } \\
\text { specialties) }\end{array}$ \\
\hline & & $\begin{array}{l}\text { Lack of respect and trust by consultants of same and other } \\
\text { specialties }\end{array}$ \\
\hline & & Dictation by surgeons \\
\hline & & Lack of specific feedback (positive or negative) \\
\hline & & $\begin{array}{l}\text { Lack of understanding among care providers in crises } \\
\text { situations. }\end{array}$ \\
\hline & \multirow[t]{3}{*}{ Institutional policies } & No accommodation for male residents \\
\hline & & No transport in strikes and riots \\
\hline & & Less time for orientation \\
\hline
\end{tabular}

awareness, stress management and conflict resolution skills as part of the generic curriculum. Counseling and mentoring should be an integral part of all training programs (Radhika et al., 2006).

Antagonistic colleagues in any multidisciplinary work performance area are a distraction from patient care and cause of tensions, cynicism and erosion of humanistic qualities (Khan \& Khimani, 2007). Communication, information sharing, collaboration and cooperation facilitates team building, empathy and a healthy organizational culture.

A number of residents enter training programs after a period of experience in Pakistan public hospitals where facilities and standards have wide variation from those in Aga Khan University. Expectations gaps of both faculty and learners need to be addressed by awareness of previous learning opportunities and provision of organizational support for addressing knowledge and competence gaps. Adherence to policies for loop closure in contentious issues is an essential area to prevent mistrust and cynicism in the learner. Realistic workloads and resolution of tensions in personal lives particularly related to financial and logistic support in times of crises is also important for quality of work and learning (Haoka et al., 2010, Boerjan et al., 2010; Businger et al., 2010). 


\section{Conclusions}

Although this study has looked into stress in only one residency program in an institution we think that this method can be used to develop a deeper understanding of the stressors in the life of residents. Tensions emerging from lack of tolerance for divergent practices of a diverse faculty, lack of discussion and dialogue, and non-threatening, non-judgemental feedback are the major workplace stressors identified by residents in this study. Residents also perceive lack of respect for junior residents outside the parent department as a major stressor affecting behaviour adversely. The stressors that have been identified in this study could be found in other programs at any other institution and the information obtained from this research is a valuable resource for evaluation of the workplace culture and the training program, as well as for planning self-development programs for faculty and residents.

\section{Reflective Critique on the Study}

Research of this nature is exploratory and may be considered intrusive by residents due to their vulnerability, which would cause withholding of important information. This was overcome by putting residents at ease about the objectives of the research, giving them the chance to lead the discussion and holding discussions in an easily accessible group, physically comfortable, and a secured area. We found that moderated focus group discussion was an appropriate tool for this purpose, facilitating the cascading or chaining effect in the group which made the data rich. We observed that residents warmed quickly to the intent of this study and were forthcoming about genuine issues; the presence of a faculty from the same department who is not a part of the Residency Committee facilitated easy but focused communication. The residents took ownership of identified themes willingly and responded to the semi-structured interviews with clarity and confidence. The sessions were held within the university for ease of access and convenience of residents. Seniors and juniors participated according to their availability and the sessions were uninterrupted. Participation of a faculty from the Department for Educational Development in the study was seen positively by the residents who felt that a neutral person will take their concerns beyond the Anesthesia Department and to the other areas of concern in the workplace environment.

\section{Beneficiaries of this study}

We believe the major beneficiaries of this study will be the trainees and faculty of our own training program as well as other training programs, as it will enable educators to reflect on the environment provided to the learner to achieve the academic qualification from the most important perspective, which is that of the learner. Medical educators in general will benefit from use of the qualitative research methodology to explore the learners' perspective of the culture in the academic organization.

\section{Take home messages}

The authors hope that the educators will take a positive view of the methods used to explore and analyse the hidden curriculum or the learning environment as the learners perceive it, rather than as the faculty perceives it. It not only helps faculty to reflect on themselves as role models and their own behaviours which determine organizational culture, but also to influence policies and address deficiencies in the organization over which they have more control than the learner.

\section{Acknowledgement}

We are highly grateful to MsHinaTejani for her invaluable contribution as transcriber and to the anesthesia residents for giving their valuable time for this study.

\section{References}

Al-Hazimi, A., Zaini, R., Al-Hyiani, A.M., Hassan, N., Gunaid, A., Ponnamperuma, G., Karunathilake, I., Roff, S., McAleer, S. \& Davis, M. (2004) Educational Environment in Traditional and Innovative Medical Schools: A Study in Four Undergraduate Medical Schools. Education for Health, 17 (2), pp.192- 203

Boerjan, M., Bluyssen, S.J., Bleichrodt, R.P., van Weel-Baumgarten E.M.\&Van Goor H. (2010) Work-related health complaints in surgical residents and the influence of social support and job-related autonomy, Med Educ, 44 (8), pp. 835-44.

Businger, A., Stefenelli, U. \& Guller U. (2010) Prevalence of burnout among surgical residents and surgeons in Switzerland, Arch Surg, 145 (10), pp. 1013-1016.

Fraser B.J. (1998) Classroom environment instruments: development, validity and applications. Learning Environments Research, 1, pp.7-33 
Gay, L.R., Mills, G.E \&\& Airasian, P. (2012) Educational Research: competencies for analysis and applications. $10^{\text {th }}$ ed. New Jersey: Pearson Publications.

Gregory, B., Collins, M.S., Jensen, M.M. \& Gooden, T.A. (2005) Chemical Dependency Treatment Outcomes of Residents in Anesthesiology: Results of a Survey, Anesth Analg, 101, pp. 1457-62.

Haoka, T., Sasahara, S., Tomotsune, Y., Yoshino, S., Maeno, T.\&Matsuzaki I. (2010). The effect of stress-related factors on mental health status among resident doctors in Japan, Med Edu, 44 (8), pp. 826-34.

Jordan, S., Cohen1, \& Patten, S. (2005) Well-being in residency training: a survey examining resident physician satisfaction both within and outside of residency training and mental health in Alberta, BMC Medical Education, 5, pp.21

Khan, F.A. \& Khimani, S. (2007) Customer focused incident monitoring in anaesthesia, Anaesthesia; 62 (6), pp. 586-590.

Khan, J.S. (2008) Evaluation of the educational environment of postgraduate surgical teaching. J Ayub Med Coll Abbottabad, 20, pp.3.

Klein, E.J., Jackson, C., Kratz, L. \& Marcuse, E.K et al. (2003), Teaching Professionalism to residents, Acad Med, 78, pp. 26-34.

Liamputtong, P. \& Ezzy, D. (2006) Qualitative Research Methods. Hong Kong: Oxford University Press.

Marieniss, D.P. (2004) Decreasing GME training stress to foster residents' professionalism.J Acad Med, 79 (9), pp. 825-31.

Marshall, N. Martin. (1996) Sampling for qualitative research Family Practice, 13, pp. 522-525.

Martini, S., Arfken, C.L., Churchill, A.\& Balon, R. (2004), Burnout comparison among residents in different medical specialties, Acad Psychiatry, 28 (3), pp. 240-242.
McCaul, C.L., Gibney, R., Magner, J., O'Keefe, D. \& Moriarty, D. (2007) Stress Levels and Quality of Life in Anesthesia Residents, A National Longitudinal Study, ASA abstract

Nyssen, A.S., Hansez1, I., Baele, P., Lamy, M. \& De Keyser, V. (2003) Occupational stress and burnout in anaesthesia, $\mathrm{Br} J$ Anaes, 90 (3), pp. 333-337.

Radhika, A., Ramanan, M.D., William, C., Taylor, M.D., Roger, B., Davis, ScD, and Russell S. Phillips., M.D. (April 2006) Mentoring Matters Mentoring and Career Preparation in Internal Medicine Residency Training, J Gen Intern Med, 21 (4), pp. 340-345.

Ratanawongsa, N., Wright, S.M. \& Carrese, J.A. (2008) Well-being in residency: Effects on relationships with patients, interactions with colleagues, performance, and motivation, Patient Education and Counseling, 72 (2), pp. 194-200.

Ratanawongsa, N., Wright, S.M. \& Carrese, J.A. (2007), Well-being in residency: a time for temporary imbalance? Medical Education, 4 (1), pp. 237-280.

Roff, S., McAleer, S., Harden, R., Al-Qahtani, M., Uddin, A.A., Deza, A.,Groenen, A. \&Primparyon, P. (1997). Development and validation of the Dundee Ready Education Environment Measure (DREEM). Medical Teacher, 19 (4), pp.295-299.

Samuel, S.E., Lawrencet, J.S. \& Schwartz H.J. et al., (1991), Investigating stress levels of residents: a pilot study, Medical Teacher, 13(1), pp. 89-92.

Thomas, N.K. Resident burnout, (2004) JAMA, 292 (23), pp. 2880-2889.

Tobin, K. \& Fraser, B. J. (eds.), 1998, Qualitative and quantitative landscapes of classroom learning environments. In B. J. Fraser and K. G. Tobin (eds.), The international handbook of science education (Dordrecht, The Netherlands: Kluwer Academic Publishers) pp.623-640.

West, C.P. \& Shanafelt, T.D. (2007) The influence of personal and environmental factors on professionalism in medical education, $B M C$ Med Educ, 7, pp.29, Published online 2007 August 30. doi: 10.1186/1472-6920-7-29.

\section{Appendix 1}

\section{Authors' definition of stress}

Stress is defined as physical, mental or emotional response which requires bodily or mental adjustment or any outside force or event that has an effect on the body or mind which may be positive or negative.

Negative stress impairs mental, physical and emotional health.

\section{Residents described perception of stress}

1. When you feel depressed; for example, time, resources, family priorities which you cannot tackle.

2. You have too many things to do in too little time that causes stress. 